\title{
Neutrophil function in pregnancy and rheumatoid arthritis
}

\author{
I P Crocker, P N Baker, J Fletcher
}

\begin{abstract}
Background-Pregnancy exerts suppressive effects on rheumatoid arthritis (RA). An attenuation in neutrophil function in late pregnancy which may explain this amelioration has previously been reported.

Objective-A longitudinal investigation of neutrophil activity in healthy pregnant women $(n=9)$ and pregnant patients with RA (n=9), compared with age matched non-pregnant patients with $R A(n=12)$ and healthy controls $(n=22)$.

Methods-Neutrophil activation was measured in response to the physiological receptor agonists, n-formyl-methionylleucyl-phenylalanine (fMLP) and zymosan activated serum (ZAS). Superoxide anion production (respiratory burst) was determined by lucigenin enhanced chemiluminescence (LUCL); secondary granule lactoferrin release by enzyme linked immunosorbent assay (ELISA); and CD11b, CD18, and CD62L expression by flow cytometric analysis.
\end{abstract}

Results-Stimulated neutrophil LUCL was significantly reduced in both pregnant women with RA and healthy pregnant women in the second (fMLP $43 \%$ and $69 \%$, ZAS $43 \%$ and $59 \%$, respectively) and third trimesters (fMLP $24 \%$ and $44 \%$, ZAS $32 \%$ and $38 \%$, respectively). Responses returned to normal within eight weeks of delivery and unstimulated levels remained unchanged throughout pregnancy. Basal and stimulated CD11b, CD18, and CD62L expression showed no variations throughout gestation for both pregnancy groups. Likewise, stimulated lactoferrin release and plasma lactoferrin remained unchanged. Certain morphological differences in RA neutrophils were highlighted by the flow cytometric analysis. Moreover, resting neutrophils and stimulated cells from patients with RA, including pregnant subjects, showed a marked increase in LUCL, but a reduction in CD11b, CD18, and CD62L. Low dose prednisolone and methylprednisolone had no effect on neutrophil parameters over the period of treatment with non-steroidal anti-inflammatory drugs.

Conclusion-The attenuation to neutrophil respiratory burst in both healthy and RA pregnancies may offer an explanation Mr Crocker

Email:

Ian.Crocker@nottingham.ac.uk

Accepted for publication

31 January 2000 this inflammatory disorder.

(Ann Rheum Dis 2000;59:555-564)
For more than a century clinical observations have highlighted an ameliorating effect of pregnancy on rheumatoid arthritis (RA). Since the first detailed account of this phenomenon published by Hench in $1938^{1}$ the beneficial effects of pregnancy on RA have been continually reaffirmed. Most authors agree that the activity of RA is significantly altered during pregnancy, with approximately $70 \%$ of patients experiencing a substantial resolution of pain, swelling, and stiffness (see review ${ }^{2}$ ). This symptomatic relief becomes apparent from the first trimester and then progresses throughout gestation, often enabling patients to reduce or completely interrupt the use of drugs. ${ }^{3}$ Unfortunately, this remission is short lived; more than $90 \%$ of the patients who improve will relapse within eight to nine months post partum and most within six weeks of delivery. ${ }^{4}$

An increasing number of theories have been proposed to explain this dramatic gestational improvement in RA, with the majority of these invoking one or more mechanisms of pregnancy induced immunosuppression. It is generally agreed that women are at greater risk of infection during pregnancy, with an increased incidence of specific intracellular infections $\left(\right.$ see review ${ }^{5}$ ). Although humoral immunity during pregnancy remains unchanged, ${ }^{6}$ a marked depression in cell mediated immunity has been suggested by diminished skin reaction to tuberculin, ${ }^{7}$ and by prolonged skin graft survival. ${ }^{8}$ A number of factors produced by the placenta and maternal tissues have been proposed to explain these effects and, recently, the disparity between maternal and fetal HLAs has been implicated. ${ }^{9}$ However, as yet, none of these explanations so far suggested, can satisfactorily explain the pattern of improvement and then relapse of RA during and after pregnancy.

Neutrophils are thought to be of central importance in the eradication of invasive pathogens, but paradoxically they are also implicated in the inflammation and tissue damage associated with connective tissue disorders. RA is characterised by an accumulation and activation of leucocytes, predominantly neutrophils, in an affected joint. It is envisaged that during the active phase of the disease, neutrophils enter the joint and become activated to secrete tissue damaging granule enzymes and reactive oxygen metabolites. There are many lines of evidence to substantiate this model of neutrophil mediated attack. Neutrophils isolated from the synovial fluid of patients with RA have been shown to exhibit biochemical properties indicating that they have been activated in vivo ${ }^{10}$; synovial fluid contains oxidatively altered components ${ }^{11}$; and 
neutrophil derived proteins, such as myeloperoxidase, are detected within joints in a molecular form, suggesting that degranulation has occurred. ${ }^{12}$

When given this overwhelming evidence for neutrophil involvement in RA, it is not difficult to imagine that any alteration in the adherence, accumulation, or activation of neutrophils in pregnancy may have beneficial effects on the outcome of the disease. Previous studies of functional differences in neutrophils during pregnancy have emphasised a reduction in chemotaxis, ${ }^{13}$ adherence, ${ }^{14}$ and microbial killing. ${ }^{15}$ Moreover, our own studies have shown a depression in neutrophil respiratory burst during pregnancy, which may also explain this ameliorating effect. ${ }^{16}$

We have previously shown a significant reduction in the receptor mediated respiratory burst activity of neutrophils from pregnant women in the third trimester of pregnancy as compared with non-pregnant controls matched for age. ${ }^{16}$ In this current study we document the pattern of changes in neutrophil function over the course of pregnancy through longitudinal observations of normal pregnant women and pregnant patients with RA. For these subjects we have compared neutrophil respiratory burst activity, secondary granule lactoferrin release, and the expression cell surface adhesion molecules, CD11b, CD18, and CD62L. These functional markers of neutrophil status were chosen to provide a comprehensive view of ex vivo and, to a limited extent, in vivo neutrophil function. In each case measurements were related to postpartum readings, and also to age and gestationally matched, normal non-pregnant women and RA patient controls.

\section{Materials and methods} SUBJECTS

Women with classical or seropositive RA (American Rheumatism Association criteria) were recruited from the rheumatology clinic of the City Hospital, Nottingham. They comprised two study groups. The first, a group of 12 non-pregnant patients with RA (mean age 29 , range 24-33) receiving non-steroidal antiinflammatory drugs (NSAIDs) but no form of second line treatment; and the second, a group of nine pregnant patients with RA (mean age 30 , range 23-38), two of whom stopped their drug treatment at the start of the pregnancy and seven of whom continued to receive low doses of prednisolone or methylprednisolone $(2.5-10 \mathrm{mg} / \mathrm{d})$. Nine women formed a pregnancy group recruited from the antenatal clinic at the City Hospital (mean age 29, range 24-35), while a further 22 healthy, nonpregnant subjects of comparable age (mean age 30 , range 24-36) were selected from the hospital staff. Of these, the non-pregnant women were not taking the oral contraceptive pill and all subjects with any pre-existing medical disorders or taking any drugs other than vitamin or iron supplementation were also excluded. Within the two pregnancy groups, three patients with RA and eight healthy women were recruited within the first trimester, and all remaining subjects were recruited in the second trimester. A sample of $30 \mathrm{ml}$ of venous blood was obtained and measurements were recorded every four weeks throughout pregnancy and at approximately six weeks post partum. There were no demographic differences between the subject groups. Informed consent was obtained before inclusion in all cases and the study was approved by the local ethical committee.

\section{PREPARATION OF NEUTROPHILS}

Venous blood samples were placed into EDTAdipotassium salt at a final concentration of 3 $\mathrm{mmol} / \mathrm{l}$. Neutrophils were isolated by a rapid, single step technique with Ficoll-Hypaque solution of $1.114 \mathrm{~g} / \mathrm{ml}$ Polyprep (Nycomed, Birmingham, UK), as described previously. ${ }^{17}$ Neutrophils were regularly obtained with a purity greater than $97 \%$ and a viability greater than $99 \%$. Cells were resuspended in sterile low endotoxin phosphate buffered saline (PBS) pH 7.2 and used immediately.

\section{STIMULATION}

The agonists used in the respiratory burst, adhesion molecule expression, and degranulation assays were $\mathrm{n}$-formyl-methionyl-leucylphenylalanine (fMLP) (Sigma Chemical Co, Poole, UK) and complement C5a des arg in zymosan activated serum (ZAS). A standard supply of ZAS was produced by the method of Fernandez et al. ${ }^{18}$ Sterile low endotoxin PBS was used as an unstimulated control.

RESPIRATORY BURST ACTIVITY

Neutrophil extracellular superoxide anion production was measured by lucigenin enhanced chemiluminescence (LUCL) using a Labsystems (Basingstoke, UK) Luminoskan plate

Table 1 Pregnancy and rheumatoid arthritis (RA) leucocyte variations. Values are means (SEM)

\begin{tabular}{lrclll}
\hline Subject group & No & $\begin{array}{l}\text { Total leucocytes } \\
\left(\times 10^{6} / \mathrm{ml}\right)\end{array}$ & $P M N \neq\left(\times 10^{6} / \mathrm{ml}\right)$ & $\begin{array}{l}\text { Lymphocytes } \\
\left(\times 10^{6} / \mathrm{ml}\right)\end{array}$ & Monocytes $\left(\times 10^{6} / \mathrm{ml}\right)$ \\
\hline Healthy non-pregnant & 22 & $5.96(0.62)$ & $3.35(0.23)$ & $1.82(0.23)$ & $0.42(0.05)$ \\
$1^{\text {st }}$ trimester pregnant & 6 & $7.32(0.68)$ & $4.88(0.64)$ & $1.84(0.27)$ & $0.44(0.04)$ \\
$2^{\text {nd }}$ trimester pregnant & 9 & $7.81(0.71)$ & $5.61(0.50)^{\star \star}$ & $1.74(0.23)$ & $0.41(0.05)$ \\
$3^{\text {rd }}$ trimester pregnant & 9 & $10.24(1.30)^{\star}$ & $7.32(1.10)^{\star \star}$ & $2.32(0.27)$ & $0.84(0.17)^{\star \star}$ \\
Post partum & 9 & $5.80(0.54)$ & $2.99(0.39)$ & $2.18(0.36)$ & $0.47(0.12)$ \\
RA non-pregnant & 12 & $8.04(0.72)^{\dagger}$ & $5.02(0.61)^{\dagger}$ & $1.94(0.23)$ & $0.43(0.07)$ \\
$1^{\text {st }}$ trimester RA & 3 & $9.80(0.92)^{\star}$ & $5.31(0.67)$ & $2.89(0.32)^{\star}$ & $0.62(0.18)$ \\
$2^{\text {nd }}$ trimester RA & 9 & $12.21(2.18)^{\star \star}$ & $7.81(1.16)^{\star}$ & $3.06(1.25)^{\star}$ & $0.63(0.20)$ \\
$3^{\text {rd }}$ trimester RA & 9 & $10.13(1.03)^{\star \star}$ & $6.28(0.68)^{\star}$ & $2.17(0.30)$ & $0.86(0.25)^{\star}$ \\
Postpartum RA & 9 & $6.94(0.41)$ & $4.12(0.44)$ & $1.83(0.24)$ & $0.42(0.05)$
\end{tabular}

Statistical significance: ${ }^{\star} \mathrm{p}=0.05$ and ${ }^{\star \star} \mathrm{p}<0.01$ compared with postpartum visit; $\mathrm{tp}<0.05$ compared with non-pregnant control group. $\ddagger \mathrm{PMN}=$ polymorphonuclear leucocytes. 
reading luminometer. Briefly, $140 \mu \mathrm{PBS} \mathrm{pH}$ 7.2 containing $1 \mathrm{mM} \mathrm{CaCl}, 0.7 \mathrm{mM} \mathrm{MgCl}_{2}$, and $0.1 \%(\mathrm{w} / \mathrm{v})$ low endotoxin bovine serum albumin (PBS/Ca/Mg/BSA), $20 \mu \mathrm{l}$ of $250 \mu \mathrm{M}$ lucigenin (bis- $N$-methylacridinium nitrate), and $20 \mu \mathrm{l}$ of neutrophil suspension $\left(1 \times 10^{7} / \mathrm{ml}\right)$ were added to triplicate wells of a 96 well Immunofluor microtitre plate (Dynatech, Billingshurst, UK). The plates were warmed in the luminometer to $37^{\circ} \mathrm{C}$ before the addition of either $20 \mu \mathrm{l}$ fMLP $(10 \mu \mathrm{mol} / \mathrm{l})$ or $20 \mu \mathrm{l}$ ZAS $(50 \%(\mathrm{v} / \mathrm{v}))$. Chemiluminescence light output was monitored every 60 seconds for 30 minutes and the integral over this period expressed as relative light units (RLUs).
ADHESION MOLECULE EXPRESSION

CD11b, CD18, and CD62L expression were determined by flow cytometry using a direct immunofluorescence technique involving fixation of the cells before antibody staining. This prefixation step prevents the upregulation of $\beta_{2}$ integrin expression as a result of sample handling and has been described in detail elsewhere. ${ }^{19}$ ZAS and fMLP were added to anticoagulated ( $3 \mathrm{mM}$ EDTA) whole blood at final concentrations of $5 \%(\mathrm{v} / \mathrm{v})$ and $1 \mu \mathrm{mol} / \mathrm{l}$, respectively. After incubation with end over end rotation at $37^{\circ} \mathrm{C}$ for 20 minutes, samples were fixed with equal volumes of $1 \%(\mathrm{w} / \mathrm{v})$ paraformaldehyde (final concentration $0.5 \%$
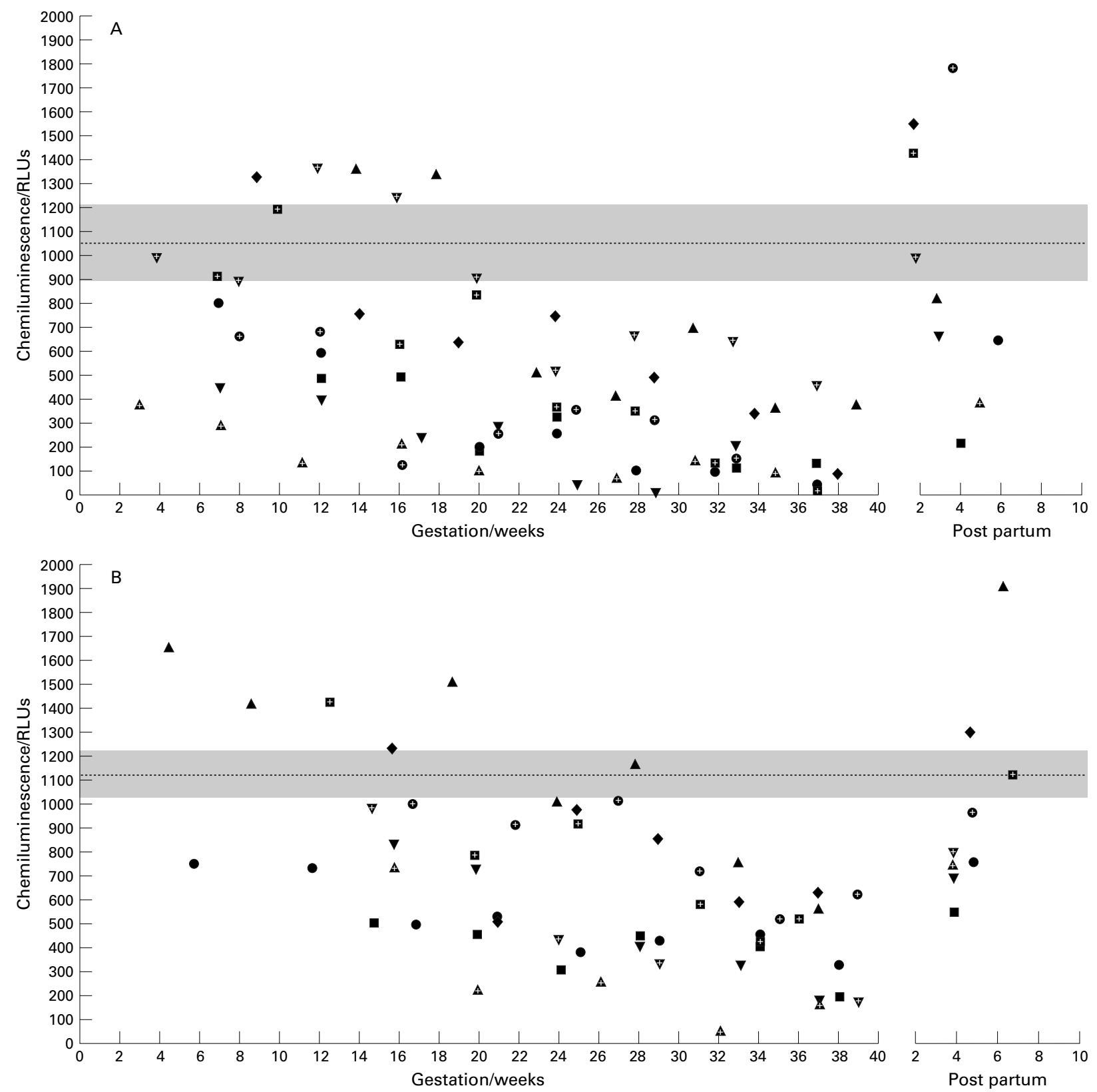

Figure 1 Basal neutrophil lucigenin enhanced chemiluminescence and the effect of $f M L P$ stimulation on cell responses from $(A)$ healthy pregnant women $(n=9)$ and non-pregnant controls matched for age $(n=22) ;(B)$ pregnant $R A$ women $(n=9)$ and non-pregnant patients with $R A$ matched for age $(n=12)$. Integral chemiluminescence light output was recorded as relative light units (RLUS). The non-pregnant results are represent as shaded areas of means (SEM). Longitudinal raw data for pregnant subjects are represented by individual symbols. Stimulated cell responses above resting cell levels are indicated. During the second and third trimester of pregnancy, respiratory burst activity was significantly reduced for both pregnancy groups in response to fMLP and zymosan activated serum (ZAS) stimulation (ZAS results not shown). Neutrophil activity for both pregnancy groups returned to their respective control levels within seven weeks of delivery (see table 2). 
$(\mathrm{v} / \mathrm{v}))$. In addition, a control tube was set up where antigen expression was fixed immediately after venesection. At the end of the 10 minute fixation period (carried out at room temperature), erythrocytes were removed by a 10 minute lysis (FACs Lysing Solution, Beckton Dickinson, Oxford, UK) and antibody staining was performed immediately by a direct immunofluorescence technique.

The antibodies used were antihuman CD11b-phycoerythrin (PE) conjugate (Becton Dickinson), antihuman CD18-fluorescein isothiocyanate (FITC) conjugate (Dako, High Wycombe, UK), and antihuman CD62LFITC conjugate (Becton Dickinson); as a negative control a combination of IgG1-FITC and IgG2a-PE antikeyhole limpit haemocyanin (Becton Dickinson) was used. As a double labelling technique was employed for the measurement of CD11b/CD18 expression it was important to set a compensation on the flow cytometer (FACScan, Beckton Dickinson); this was done by setting up an additional control tube containing FITC-anti-CD3 and PE-anti-CD19, markers for $\mathrm{T}$ and $\mathrm{B}$ lymphocytes respectively. The compensation was set by creating a live gate around the lymphocyte population and electronically compensating the FITC and PE fluorescences to correct for spectral overlap. As an instrument control measure, CaliBRITE beads (Becton Dickinson) were used before each run to ensure the correct alignment and calibration of the flow cytometer. The day to day variation was found to be $<0.1 \%$.

MEASUREMENT OF LACTOFERRIN

Lactoferrin in either plasma or secreted from stimulated neutrophils was measured by an enzyme linked immunosorbent assay (ELISA) as previously described. ${ }^{17}$ Whole blood leucocytes, obtained after erythrocyte lysis $(0.83 \%$ $\mathrm{NH}_{4} \mathrm{Cl}, 10$ minutes), were incubated in sterile PBS pH 7.2 containing $0.1 \%(\mathrm{w} / \mathrm{v})$ BSA (PBS/ BSA) with either PBS as control, $1 \mu \mathrm{M}$ fMLP, or $5 \%(\mathrm{v} / \mathrm{v})$ ZAS in a final volume of $500 \mu \mathrm{l}$. Cells were incubated for 30 minutes at $37^{\circ} \mathrm{C}$ with end over end rotation, after which they were centrifuged at $400 \times g$ for 5 minutes. The supernatant was removed and stored at $-70^{\circ} \mathrm{C}$ for measurement of secreted lactoferrin. As pregnancy is considered to be a state of leucocytosis, ${ }^{20}$ and neutrophils the sole source of circulating lactoferrin, ${ }^{21}$ whole blood counts were carried out to determine the amount of lactoferrin for a standard number of cells. Plasma levels and neutrophil lactoferrin release were expressed as picomoles per $10^{6}$ neutrophils based on the number of cells in the leucocyte differential counts.

\section{STATISTICAL ANALYSIS}

Trends over the course of each pregnancy were determined by linear regression analysis. Each subject's regression coefficient was combined by study group - that is, healthy pregnant or RA pregnant, and then compared by an independent sample Student's $t$ test. For further statistical analysis, single data points were selected from multiple readings for each subject within the following periods of each trimester: 5-9 weeks, 18-22 weeks, 32-36 weeks, and for 4-8 weeks post partum. For repeated readings, selections were restricted to those nearest to the middle of each time period. The reduced numbers of data points in the first trimesters prevent their inclusion in this statistical analysis, but these measurements are included in the results section. Statistical significance of difference for normally distributed data was determined using either a one way analysis of variance test or Student's $t$ test (with or without Bonferroni correction). Normal distribution was assessed by the Shapiro-Wilk significance level normality test. The results are presented as means and standard errors of the means (SEM), with the data considered significant at $\mathrm{p}<0.05$.

\section{Results}

PREGNANCY AND RA HAEMATOLOGICAL

VARIATIONS

Table 1 shows the changes in leucocyte differential blood counts over the course of pregnancy in healthy and RA women as compared with postpartum and non-pregnant controls. During normal pregnancy there was a progressive increase in total leucocyte counts, mainly owing to an increase in the number of circulating neutrophils but also, to a lesser extent, an increase in monocyte numbers in the third trimester. A similar increase in total cell numbers, neutrophils, and monocytes occurred in RA pregnancies, but also with an increase in lymphocytes during the first and second trimesters. All variables returned to control levels after delivery, but baseline numbers of

Table 2 Receptor stimulated neutrophil lucigenin enhanced chemiluminescence. Values are means (SEM) relative light units (RLU)

\begin{tabular}{|c|c|c|c|c|}
\hline \multirow[b]{2}{*}{ Stimulus } & \multirow[b]{2}{*}{ Healthy non-pregnant } & \multicolumn{3}{|c|}{ Healthy pregnant (trimesters) } \\
\hline & & 2 & 3 & Post partum \\
\hline \multirow{3}{*}{$\begin{array}{l}\text { fMLP† } \\
\text { ZAS } †\end{array}$} & $1272(226)$ & $621(141)(\mathrm{p}<0.05)^{\star}$ & $342(71)(\mathrm{p}<0.05)^{\star \star}$ & 1440 (440) (NS) \\
\hline & $1134(258)$ & $\begin{array}{l}519(135)(\mathrm{p}<0.05)^{\star \star} \\
R A \text { pregnant (trimesters) }\end{array}$ & $383(72)(\mathrm{p}<0.01)^{\star \star}$ & $1202(190)(\mathrm{NS})$ \\
\hline & $R A$ non-pregnant & 2 & 3 & Post partum \\
\hline fMLP & $1558(115)$ & $863(121)(\mathrm{p}<0.03)^{\star \star}$ & $550(53)(\mathrm{p}<0.01)^{\star \star}$ & 1248 (201) (NS) \\
\hline ZAS & $1636(76)$ & $958(94)(\mathrm{p}<0.01)^{\star \star}$ & $621(93)(\mathrm{p}<0.01)^{\star \star}$ & $1626(200)$ (NS) \\
\hline
\end{tabular}

One way analysis of variance analysis; healthy pregnant subjects (fMLP $\mathrm{p}<0.03$, ZAS $\mathrm{p}<0.01$ ), pregnant subjects with RA (fMLP $\mathrm{p}<0.01$, ZAS $\mathrm{p}<0.01)$. Statistical significance; bracketed $\mathrm{p}$ values compared with non-pregnant controls, ${ }^{\star} \mathrm{p}<0.05$ and ${ }^{\star \star} \mathrm{p}<0.01$ compared with postpartum measurements.

†fMLP = n-formyl-methionyl-leucyl-phenylalanine; ZAS = zymosan activated serum. 
neutrophils were originally higher in the patients with RA than in their equivalent healthy controls.

EX VIVO SUPEROXIDE ANION PRODUCTION

In response to the receptor mediated stimuli, fMLP and ZAS, the respiratory burst activity in neutrophils isolated from healthy pregnant women in the second and third trimesters was significantly reduced compared with postpartum measurements and non-pregnant controls
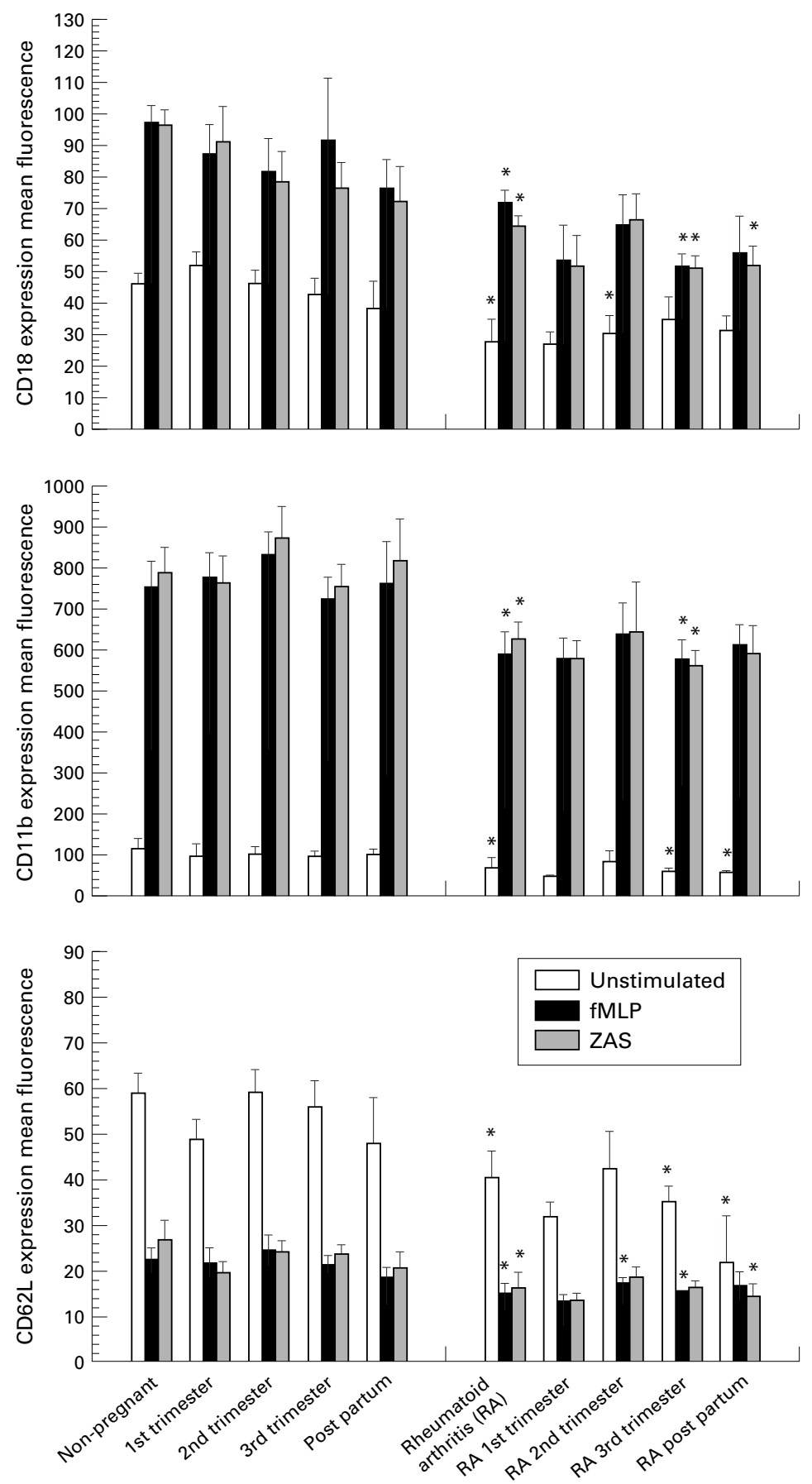

Figure 2 Expression of adhesion molecules on the surface of neutrophils from the four study groups. The results are given for $C D 11 b, C D 18$, and $C D 62 \mathrm{~L}$ and show changes in mean fluorescence units before stimulation (phosphate buffered saline) and after stimulation with $f M L P$ or zymosan activated serum. Data are expressed as mean values (SEM). Statistical significance of difference was determined between the rheumatoid groups and their equivalent healthy, non-pregnant and pregnant control groups. The degree of statistical significance is indicated by $\star_{p}<0.05$ and ${ }^{\star} p<0.01$ (t test). (fig 1, table 2). Similarly, responses of patients with RA showed a reduction in respiratory burst over the course of the pregnancy and a return to control levels after delivery (fig 1, table 2). Analysis of the first trimester was excluded because of lower sample numbers. One-way analysis of variance of the remaining trimesters highlighted a progressive reduction in neutrophil responses to fMLP and ZAS, in both healthy pregnant and RA pregnant women. This trend over the course of each pregnancy was further compared by regression analysis, combining the regression coefficients for each pregnant group. The results confirmed no differences between healthy and RA pregnant women in their rates of reduction in neutrophil respiratory burst. There were no significant differences between any postpartum responses and controls, but it was recognised that a significant increase in basal levels of resting cells did exist in all cases between pregnant and non-pregnant patients with RA, and their equivalent healthy controls (statistics not shown).

\section{ADHESION MOLECULE EXPRESSION}

The use of a rapid leucocyte fixation and preparation procedure allowed for the determination of the unstimulated expression of CD11b, CD18, and CD62L immediately after venesection. The results can be presented as either mean log fluorescence units or as the percentage of neutrophils positive for the fluorescence antibody. Neutrophil CD11b and CD18 expression were increased by activation with chemotactic agents (fig 2). Conversely, CD62L is down-regulated during activation and responded to stimulation by a significant decrease in percentage positive cells (fig 3) and mean fluorescence units (fig 2). For CD11b and CD18 expression the mean fluorescence measurements are given for positively stained neutrophils. This population routinely contained $98 \%$ of all neutrophils counted. As shown in fig 2 there were no differences in the unstimulated or stimulated expression of CD11b, CD18, or CD62L between the normal non-pregnant measurements and the normal pregnant and postpartum values. Similar results were recorded for RA pregnant and nonpregnant groups, showing no differences in both stimulated and unstimulated expression for all three adhesion molecules studied. In addition, neutrophils from non-pregnant RA women expressed less CD11b, CD18, and CD62L overall than their equivalent controls. However, when presented as the percentage of cells expressing CD62L there was no significant difference between healthy controls and patients with RA, whether pregnant or not (fig 3). The fixation method used had no effect on adhesion molecule expression over the experimental time period (data not shown).

LACTOFERRIN RELEASE

Direct measurements of plasma lactoferrin concentrations showed a significant difference between the healthy and RA non-pregnant groups (2.55 (0.41) nmol/1 and 3.95 (0.58) $\mathrm{nmol} / 1$ ( $\mathrm{p}<0.01)$, respectively; fig 4$)$. However, 
when values were normalised to account for the number of circulating neutrophils, equivalent readings were achieved (fig 5). For measurements of normal pregnant versus RA pregnant whole blood cells, the values of lactoferrin released in response to fMLP and ZAS showed variations between trimesters and also between subject groups (fig 4). Again these differences were abolished when the number of lactoferrin producing neutrophils present were accounted for and the measurements were expressed as lactoferrin released ( $\mathrm{pmol} / 10^{6}$ cells; fig 5).
LIGHT SCATTERING PROPERTIES

The flow cytometer allows the visual representation of cell populations according to their light scattering properties. For neutrophils, a forward and side scatter value will give a crude but measurable indication of both cell size and cell complexity. Therefore, morphological changes during neutrophil maturation and activation can be evaluated, at least to a limited degree, by the assessment of these light scattering characteristics. For example, an increase in forward scatter and a concomitant decrease in

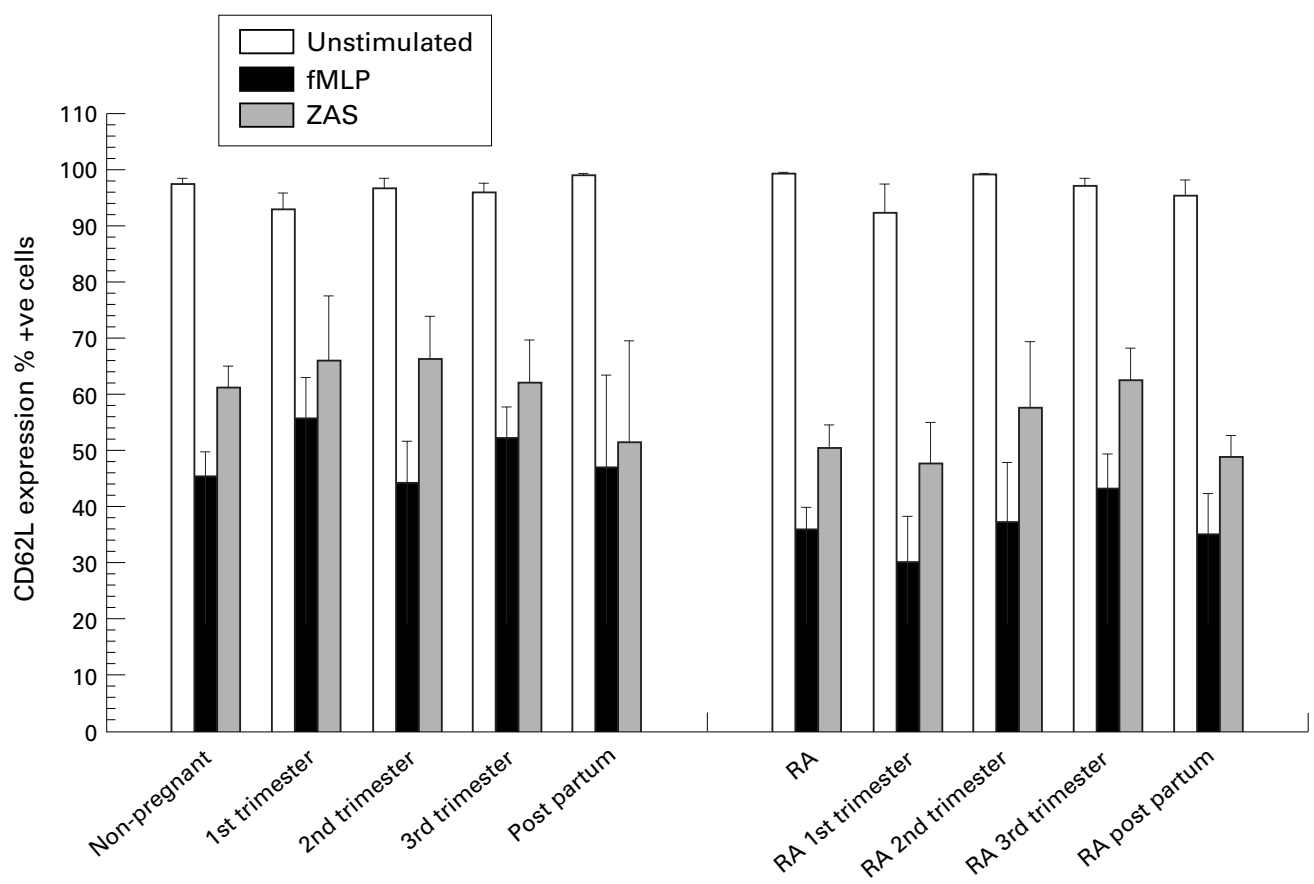

Figure 3 Expression of CD62L on the surface of neutrophils from women of the four study groups. The results show changes in the percentage of positive cells present before stimulation (phosphate buffered saline) and after stimulation with fMLP or zymosan activated serum. Data are expressed as mean values (SEM).

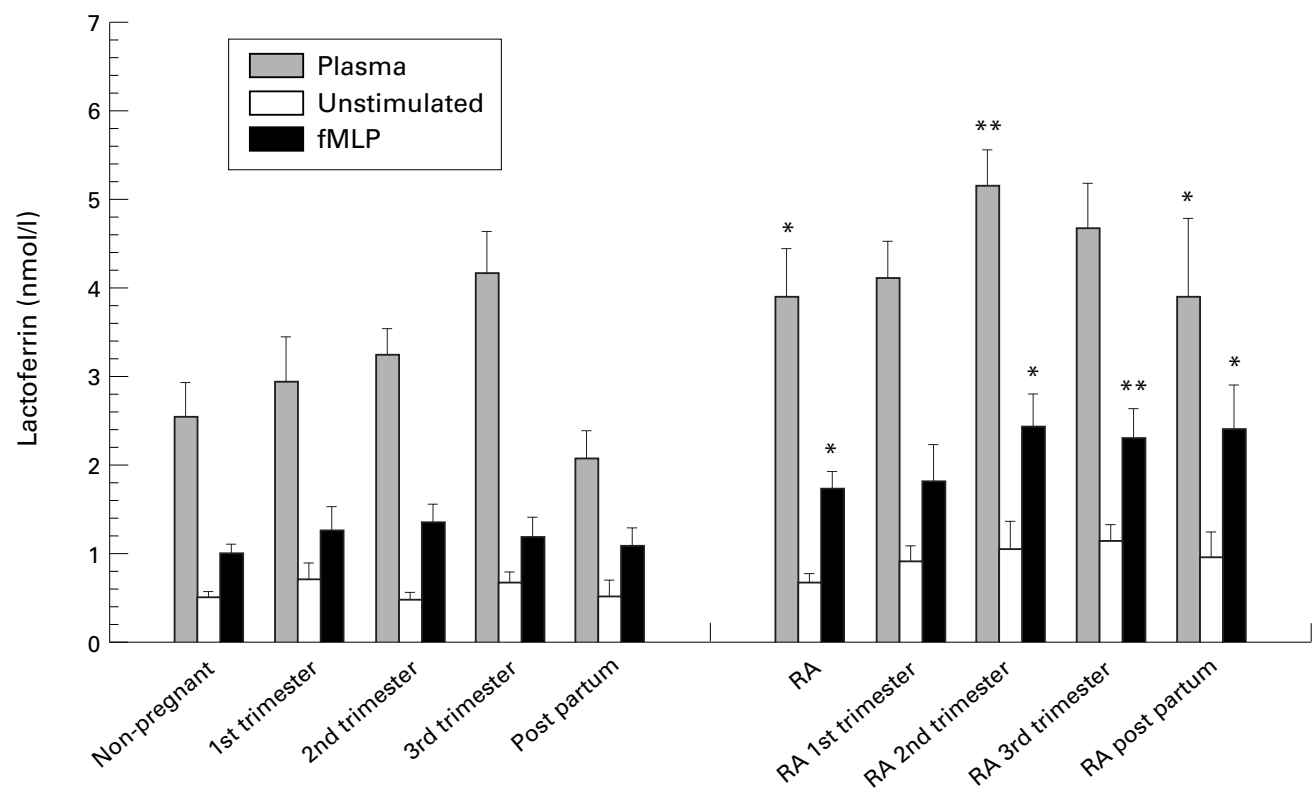

Figure 4 Plasma lactoferrin and unstimulated (phosphate buffered saline) and stimulated (fMLP, zymosan activated serum) whole blood lactoferrin release from non-pregnant and pregnant healthy women, and from non-pregnant and pregnant patients with $R A$ matched for age. Data are shown as mean (SEM) concentrations (nmol/l). Statistical significance of difference was determined between the $R A$ groups and their equivalent healthy, non-pregnant and pregnant control groups. The degree of significance is indicated by ${ }^{\star} p<0.05$ and ${ }^{\star *} p<0.01$ (t test). 


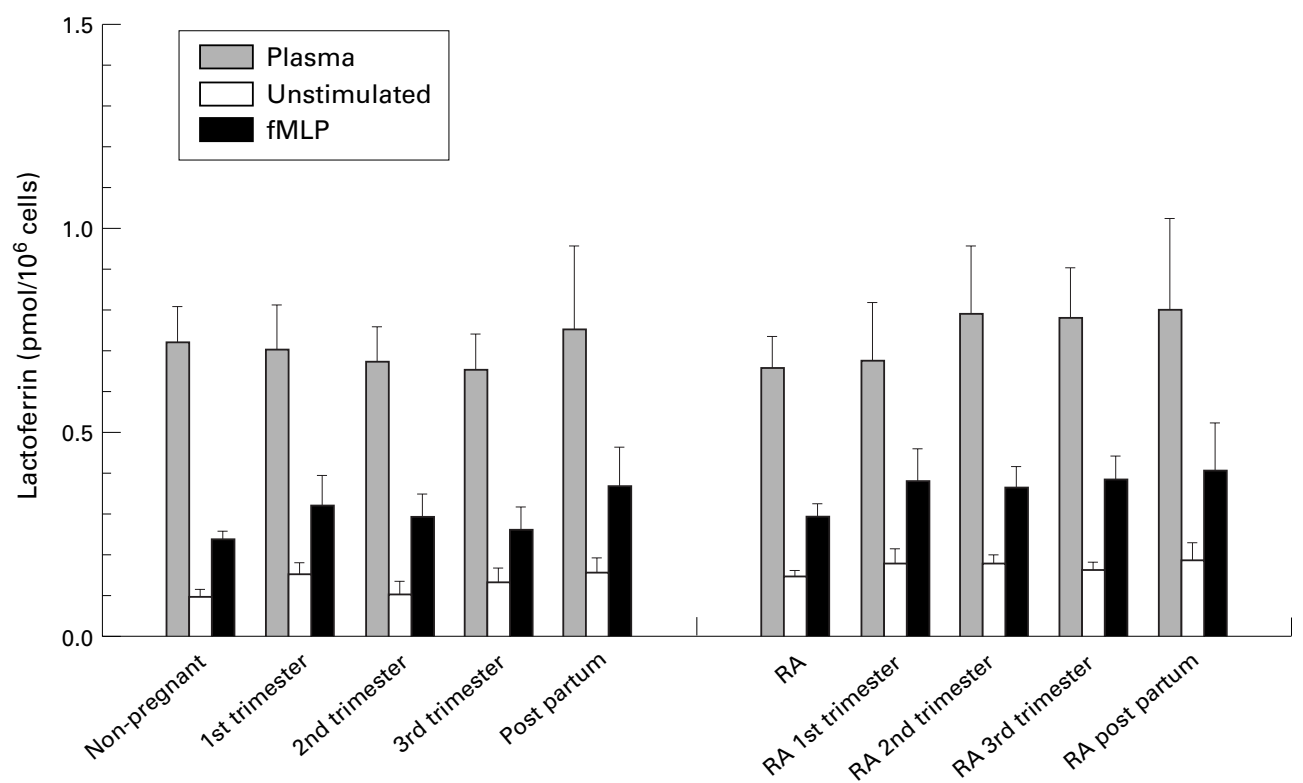

Figure 5 Lactoferrin concentrations normalised to $10^{6}$ cells for plasma and unstimulated (phosphate buffered saline) and stimulated neutrophils ( $\mathrm{MLP}$, zymosan activated serum) of healthy non-pregnant and pregnant women and RA, non-pregnant and pregnant women. The data are expressed as means (SEM) (pmol/10 cells).

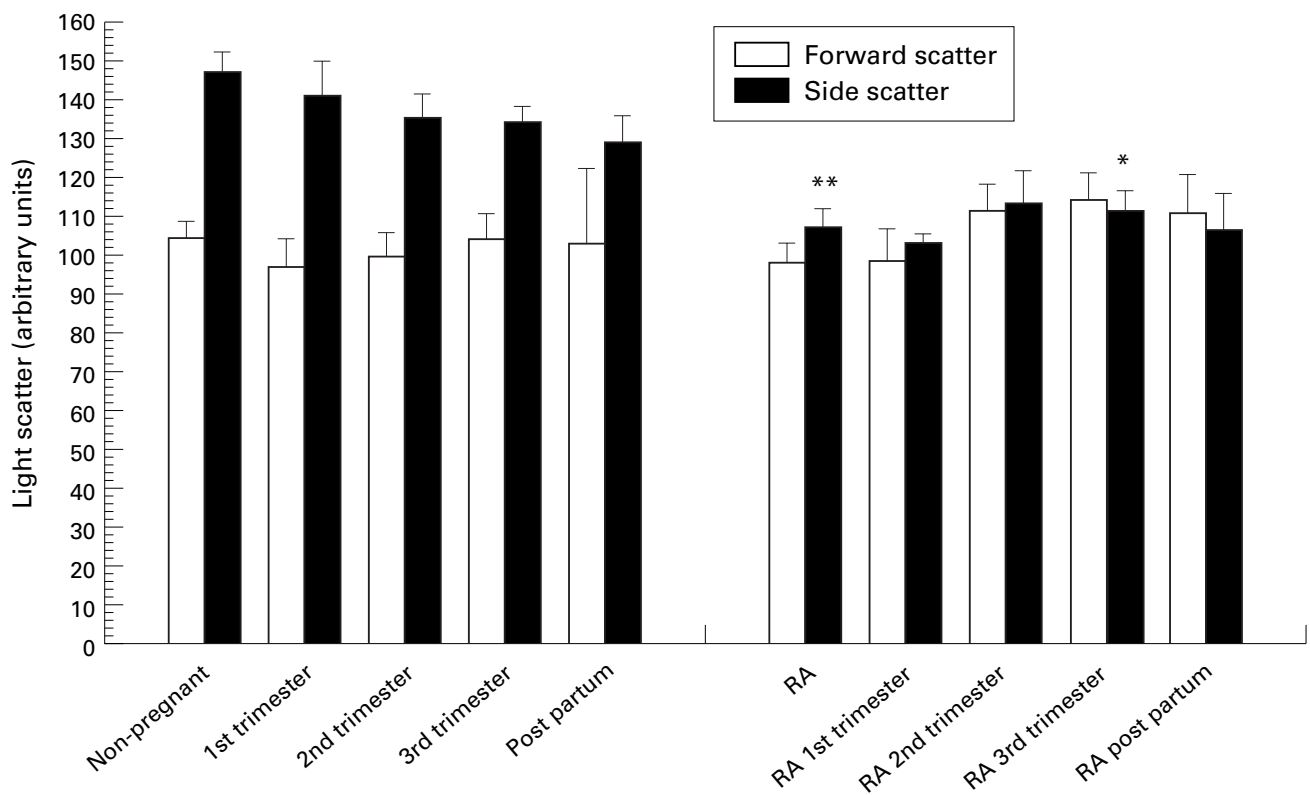

Figure 6 Morphological differences for neutrophils from healthy non-pregnant and pregnant women and $R A$ non-pregnant and pregnant women as assessed by their light scattering properties on a flow cytometer. Forward scatter indicates cell size and side scatter shows cell complexity. The data are expressed as mean values (SEM). Statistical significance of difference was determined between the rheumatoid groups and their equivalent healthy, non-pregnant and pregnant control groups. The degree of significance is indicated by ${ }^{\star} p<0.05$ and ${ }^{\star} x_{p}<0.01$ ( $t$ test).

side scatter readings are always observed when cells undergo degranulation, either through "priming" or activation by an appropriate stimulus. In the case of our four study groups the mean forward scatter intensity, representative of cell size and dimensions, showed no differences between cells from both RA and/or pregnant states. However as fig 6 indicates, side scatter analysis of these same cells did show a decrease in cell complexity for the RA group compared with the non-pregnant controls. Although paraformaldehyde fixation can affect neutrophil light scattering properties all cells were fixed and treated in the same way. Therefore, this observation may be highlighting a morphological difference in the neutrophils of patients with RA, one which persists throughout all stages of pregnancy.

\section{Discussion}

We have previously shown alterations to neutrophil function during healthy pregnancy. The objective of this study was to confirm whether these changes occur in pregnant patients with RA, and therefore provide an explanation for the ameliorating affects of pregnancy on RA. To achieve this objective we investigated neutrophil respiratory burst activity, adhesion molecule expression, and lactoferrin release in pregnant 
and non-pregnant patients with RA and healthy subjects.

Neutrophil respiratory burst activity was measured in response to physiological stimuli using lucigenin, a chemiluminescent probe selective for extracellular superoxide anions. ${ }^{22}$ Our findings suggest that although basal neutrophil responses remain unaltered, stimulated respiratory burst activity is diminished throughout the course of pregnancy. This decline in activity is characterised by a gradually depression from the second trimester followed by a return to preconception and control levels within eight weeks of delivery. Although consistent with earlier studies of superoxide production, these results are in conflict with studies employing less direct measurements of respiratory burst. For instance, studies using luminol chemiluminescence have shown a trend towards increased respiratory burst activity during pregnancy, ${ }^{23}$ whereas those employing more selective measurements of superoxide anions, such as lucigenin, have shown a consistent reduction. ${ }^{24} \mathrm{~A}$ previous in vitro observation by Buyon et al has highlighted the inhibitory affect of oestradiol on neutrophil superoxide generation. ${ }^{25}$ In light of these findings, and in support of this study, it must be emphasised that the timing of the diminished responses to fMLP and ZAS is consistent with reported clinical observations of reduced symptomatic pain relief in RA. ${ }^{3}$ It must also be recognised that these results are in agreement with an inhibitory effect of pregnancy sera on the phagocytosis of viable bacteria, ${ }^{26}$ and also with the increased incidence of specific intracellular infections during the latter part of gestation. ${ }^{5}$

In addition to the ability of neutrophils to become activated in response to inflammatory stimuli, it is widely recognised that bloodstream neutrophils can also be primed by suboptimal concentrations of stimuli, or by exposure to relevant inflammatory cytokines. More simply, any form of priming in vivo, or in vitro, results in molecular modifications to neutrophils and an accompanying enhancement in their functional responsiveness. In RA, an increase in the levels of cytokines has been identified through a rise in the circulating concentrations of tumour necrosis factor $\alpha$ and interlukin $6 .^{27}{ }^{28}$ Although these cytokines are enhancers of stimulated neutrophil lucigenin chemiluminescence in vitro, ${ }^{29}{ }^{30}$ we have previously reported a decline in tumour necrosis factor $\alpha$ and interleukin priming of neutrophils from the third trimester of pregnancy. ${ }^{16}$ The new data from this study indicate that the resting cell responses for healthy pregnant and non-pregnant subjects remain consistent, but for patients with RA these same cell responses are considerably raised. Although this might represent a partial "priming" of bloodstream neutrophils in the disease state, the observed alterations in neutrophils during pregnancy are nevertheless reaffirmed. Pregnant patients with RA showed the same reduction in respiratory burst and subsequent return to non-pregnant control levels as those of equivalent healthy pregnancy cells.
In RA, an extensive granulocytic infiltration of the pannus and periarticular tissues, coupled with the prevalence of proinflammatory cytokines and immune complexes in the synovial fluid (SF), provides a microenvironment compatible with neutrophil activation. In support of this inflammatory model, preparations of neutrophils isolated from affected joints show convincing evidence of in vivo activation. ${ }^{31}$ In addition to RA, it has also been suggested that circulating neutrophils in pregnancy are activated and as a consequence show reduced phagocytosis ex vivo. ${ }^{32}$ Experimentally, neutrophil priming and activation can be best identified by a transformation in cellular adhesion molecule expression - most notably by a rise in the $\beta_{2}$ integrin, CR3 (CD18/CD11b) expression, and a fall in L selectin, CD62L. The $\beta_{2}$ integrins are up-regulated on the cell surface through degranulation, ${ }^{33}$ whereas CD62L is constitutively expressed and is shed upon cell activation/priming by proteolytic cleavage. ${ }^{34}$ Our data show that although the expression of CD18, CD11b, and CD62L on unstimulated neutrophils is unaltered in pregnancy, differing observations are evident for pregnant and nonpregnant patients with RA. No marked differences in the patterns of responsiveness are apparent in the stimulated responses of both pregnant and non-pregnant RA groups, but basal levels of expression in RA of all adhesion molecules examined were significantly lower. Although a surprising observation, which conflicts with other studies, ${ }^{35}{ }^{36}$ similar results have previously been reported. ${ }^{37} 38$

A possible clue to this expression of adhesion molecules is given by the light scattering properties of RA cells within the flow cytometer. The measured decrease in side scatter, and absence of an accompanying forward scatter transformation, is a new finding for neutrophils. Although unusual and open to interpretation, this observation strongly implies morphologically different neutrophils in pregnant and non-pregnant patients with RA.

Lactoferrin, a widely distributed protein, stored predominantly in the specific granules of neutrophils, has been shown to be released during normal neutrophil activation in vitro. ${ }^{39}$ Together with elastase-a proteolytic enzyme stored in the azurophil granules - high concentrations of lactoferrin are evident in affected joints, ${ }^{40} 41$ and raised circulating levels have been shown in RA. ${ }^{42}$ Strong correlations with elastase-proteinase inhibitor complex and C reactive protein measurements of disease activity have advanced lactoferrin as a possible marker for neutrophil dependent inflammation in RA. This study has shown increased levels of plasma lactoferrin in RA and the latter stages of pregnancy, and has also highlighted an increase in lactoferrin upon whole blood receptor stimulated release.

Although on first inspection these results may indicate prior activation in vivo and a primed responsiveness of cells in vitro, the authors believe a cautionary note must be added to this interpretation. In the past, both plasma lactoferrin in patients with cyclic neutropenia, ${ }^{43}$ and plasma and SF lactoferrin 
and elastase in patients with $\mathrm{RA},{ }^{42}$ have all been correlated significantly with absolute neutrophil counts. In fact, when our results are corrected for circulating lactoferrin producing neutrophils, no discernible differences in plasma measurements or cellular responses could be established between the study groups. It may be concluded that for this study the raised plasma levels in pregnancy and RA are a direct reflection of neutrophil numbers and turnover and not, as previously assumed, a result of either cellular degranulation or activation in vivo.

Although the functional status of SF derived neutrophils is difficult to dispute, it is currently contentious whether full or partial activation occurs in circulating neutrophils of patients with RA. Previous studies claim that certain drugs used in the treatment of RA may moderate peripheral blood neutrophil numbers and responsiveness. ${ }^{44-46}$ Certainly, glucocorticoid treatments will raise neutrophil numbers, but their ability to alter cellular function remains undetermined. In vitro observations suggest that neutrophils are largely resistant to therapeutic levels of methylprednisolone ${ }^{47}$ and other antirheumatic drugs. ${ }^{48}$ However, similar in vivo studies suggest a decrease in neutrophil ingress into joints ${ }^{49}$ and an associated decrease of CD $11 \mathrm{~b}$ and CD $18 .^{50}$ Obviously, the recruitment of subjects with active RA who are not taking drugs is difficult, and this becomes even more confounded when restricted to women of childbearing age. In this study the combination of treatments and groups allows some conclusions to be drawn. The postpartum readings of the pregnant RA group provide a direct comparison between two sets of non-pregnant patients with RA, one group taking NSAIDs and the other methylprednisolone/ prednisolone. As differences between these groups were not identified, it can be said that prednisolone has no affect on neutrophils above that offered by NSAIDs. Given that the action of glucocorticoids and NSAIDs on neutrophil function remains debatable, a full interpretation of their effects is difficult. These agents may alter adhesion molecule expression, but it seems unlikely that the neutrophil respiratory burst will be affected by both these treatments. Similarly, glucocorticoids may explain the raised neutrophil levels in the patients with RA studied, but NSAID treated subjects, as well as those taking prednisolone, also showed raised neutrophil numbers. As NSAID treatment is unlikely to affect neutrophil numbers, a more plausible explanation for this and other observations is that changes in neutrophil behaviour are a consequence of disease activity rather than drug treatment.

In this study the pattern of adhesion molecule expression on neutrophils from patients with RA suggests morphologically different cells with functionally normal or even primed responses. Moreover, similarities in cellular activities and adhesion molecule markers indicate that any proposed in vivo activation or priming of neutrophils does not occur in normal pregnancy. The observed reduction of neutrophil superoxide anion release in preg- nant patients with RA occurs regardless of their drug treatment and is a particularly interesting observation. Superoxide anions act as precursors for a number of reactive oxygen species that are thought to be important in degrading protective proteins and directly contributing to tissue destruction. Undoubtedly, any pregnancy associated depression of neutrophil function might conceivably reduce the degree of SF inflammation and thereby contribute to the improvement of RA. Currently, efforts are underway to establish a rationale for the observed changes in the respiratory burst of neutrophils in pregnancy.

1 Hench PS. The ameliorating effect of pregnancy on chronic atrophic (infectious rheumatoid) arthritis, fibrositis, and intermittent hydrarthrosis. Proceedings of Staff Meetings Mayo Clinic 1938;45:161-7.

2 Spector TD, DA Silva JAP. Pregnancy and rheumatoid arthritis: an overview. Am J Reprod Immunol 1992;28: 222-5.

3 Da Silva JAP, Spector TD. The role of pregnancy in the course and etiology of rheumatoid arthritis. Clin Rheumatol 1992;11:189-94.

4 Perselin RH. The effect of pregnancy on rheumatoid arthritis. Bull Rheum Dis 1977;27:922-7.

5 Brabin BJ. Epidemiology of infection in pregnancy. Rev Brabin BJ. Epidemiology of
Infect Dis 1985;7:579-603.

6 Finn R, St Hill CA, Govan AJ. Immunologic response in pregnancy and survival of the fetal homograft. BMJ 1972; iii: $150-2$.

7 Andresen RH, Monroe GW. Experimental study of the behavior of adult human skin homografts during pregnancy. Am J Obstet Gynecol 1962;84:1096-103.

8 Stanworth DR. A possible immunochemical explanation for pregnancy associated remissions in rheumatoid arthritis? Ann Rheum Dis 1988;47:89-90.

9 Nelson JL, Hughes KA, Smith AG, Nisperos BB, Branchaud AM, Hansen JA. Maternal-fetal disparity in HLA class II alloantigens and the pregnancy-induced amelioration of rheumatoid arthritis. N Engl J Med 1993;12:46671 .

10 Nurcombe HL, Bucknall RC, Edwards SW. Neutrophils solated from the synovial fluid of patients with rheumatoid rthritis: priming and activation in vivo. Ann Rheum Dis 1991;50:147-53.

11 Merry P, Winyard PG, Morris CJ, Grootvel M, Blake DR. Oxygen free radicals, inflammation and synovitis: the current status. Ann Rheum Dis. 1989;48:864-79.

12 Edwards SW, Hughes V, Barrow J, Bucknall R. Immunological detection of myeloperoxidase in synovial fluid from patients with rheumatoid arthritis. Biochem J 1988;250: $81-5$.

13 Krause PJ, Ingardia CJ, Pontius LT, Malech HL, Lobello TM, Maderazo EG. Host defence during pregnancy: neutrophil chemotaxis and adherence. Am J Obstet Gynecol. 1987; 157:274-80.

14 Bjorksten B, Soderstrom T, Damber M-G, Von Schoultz, Stigbrand T. Polymorphonuclear leucocyte function during pregnancy. Scand J Immunol 1978;8:257-62.

15 El-Maallem H, Fletcher J. Impaired neutrophil function and myeloperoxidase deficiency in pregnancy. Br J Haematol $1980 ; 44: 375-81$.

16 Crouch SPM, Crocker IP, Fletcher J. The effect of pregnancy on polymorphonuclear leukocyte function. J Immunol 1995;155:5436-43.

17 Crouch SPM, Fletcher J. Effect of ingested pentoxifylline on neutrophil superoxide anion production. Infect Immun 1992;60:4504-9.

18 Fernandez HN, Henson PM, Otani A, Hugli TE. Chemotactic response to human $\mathrm{C} 3 \mathrm{a}$ and $\mathrm{C} 5 \mathrm{a}$ anaphylatoxins. I. Evaluation of $\mathrm{C} 3 \mathrm{a}$ and $\mathrm{C} 5 \mathrm{a}$ leukotaxis in vitro and under Evaluation of C3a and C5a leukotaxis in vitro and
stimulated conditions. J Immunol 1978;120:109-15.

19 Turzanski J, Crouch SP, Fletcher J, Hunter A. Ex vivo neutrophil function in response to three different doses of glycosylated rHuG-CSF (Lenograstim). Br J Haematol 1997; 96:46-54.

20 Barden A, Graham D, Beilin LJ, Ritchie J, Baker R, Walters $\mathrm{BN}$, et al. Neutrophil CD11b expression and neutrophil activation in pre-eclampsia. Clin Sci 1997;92:37-44.

21 Baynes RD, Bezwoda WR, Khan Q, Mansoor N. Relationship of plasma lactoferrin content to neutrophil regeneration and bone marrow infusion. Scand J Haematol 1986;36:79-84

22 Gyllenhammer $H$. Lucigenin chemiluminescence in the assessment of neutrophil superoxide anion production. J Immunol Methods 1987;97:209-12.

23 Selvaraji RJ, Sbarra AJ, Thomas GB, Cetrulo CL, Mitchell GW. A microtechnique for studying chemiluminescence response of phagocytes using whole blood and its applicaresponse of phagocytes using whole blood and its applica-
tion to the evaluation of phagocytes in pregnancy. Journal tion to the evaluation of phagocytes in pregnancy. J
of the Reticuloendothelial Society 1982;31:3-6.

24 Cotton DJ, Seligmann B, Obrien WF, Gallin JI. Selective defect in human neutrophil superoxide anion generation 
elicited by the chemoattractant $\mathrm{n}$-formylmethionylleucylphenylalanine in pregnancy. J Infect Dis 1983;148. leucylp

25 Buyon JP, Korchak HM, Rutherford LE, Ganguly M, Weissmann G. Female hormones reduce neutrophi responsiveness in vitro. Arthritis Rheum 1984;27:623-30.

26 Persellin RH, Thoi LL. Human polymorphonuclear leukocyte phagocytosis in pregnancy: development of inhibition during gestation and recovery in the post partum period. Am J Obstet Gynecol 1979;134:250-5.

27 Manicourt DH, Triki R, Fukuda K, Devogelaer JP, Nagant de Deuxchaisnes C, Thonar EJ. Levels of circulating tumor necrosis factor alpha and interleukin-6 in patients with rheumatoid arthritis. Relationship to serum levels of hyaluronan and antigenic keratan sulfate. Arthritis Rheum 1993;36:490-9.

28 Zangerle PF, De Groote D, Lopez M, Meuleman RJ, Vrindts Y, Fauchet F, et al. Direct stimulation of cytokines (IL-1 beta, TNF-alpha, IL-6, IL-2, IFN-gamma and GM-CSF) in whole blood: II. Application to rheumatoid arthritis and osteoarthritis. Cytokine 1992;4:568-75.

29 Baggiolini M, Walz A, Kunkel SL. Neutrophil-activating peptide-1/interleukin 8 , a novel cytokine that activates neutrophils. J Clin Invest $1989 ; 84: 1045-9$.

30 Berkow RL, Wang D, Larrick JW, Dodson RW, Howard JH. Enhancement of neutrophil superoxide production by preincubation with recombinant human tumor necrosis factor. J Immunol 1987;139:3783-91.

31 Emery P, Lopez AF, Burns GF, Vadas MA. Synovial fluid neutrophils of patients with rheumatoid arthritis have membrane antigen changes that reflect activation. Ann Rheum Dis 1988;47:34-9.

32 Miller C, Russell AS. Polymorphonuclear leukocyte response to stimulation

33 Bainton DF, Miller LJ, Kishimoto TK, Springer TA. Leukocyte adhesion receptors are stored in peroxidasenegative granules of human neutrophils. J Exp Med 1987; negative granules

34 Spertini O, Kansas GS, Munro M, Griffin JD, Tedder TF. Regulation of leukocyte migration by activation of the leukocyte adhesion molecule (LAM-1) selectin. Nature 1991;349:691-5.

35 Felzmann T, Gadd S, Majdic O. Analysis of functionassociated receptor molecules on peripheral blood and synovial fluid granulocytes from patients with rheumatoid and reactive arthritis. J Clin Immunol 1991;11:205-12.

36 Macey MG, Wilton JM, Carbon R, Edmonds S, Perry JD, $\mathrm{McCarthy} \mathrm{D}$. Leukocyte activation and function-associated antigens in inflammatory disease. Agents Actions 1993; 38(spec No):C39-40.

37 Jones J, Laffafian I, Cooper AM, Williams BD, Morgan BP. Expression of complement regulatory molecules and other surface markers on neutrophils from synovial fluid and blood of patients with rheumatoid arthritis. Br J Rheumatol 1994;33:707-12.

38 Bond A, Hay FC. L-selectin expression on the surface of peripheral blood leukocytes from rheumatoid arthritis patients is linked to disease activity. Scand J Immunol 1997;46:312-16.

39 Esaguy N, Aguas AP, Vilanova M, Silva MT. Activation of human neutrophils by phorbol ester decreases the cytoplasm compactness and the lactoferrin content of the granulocytes. F Leukoc Biol 1991;50:444-52.

40 Malmquist J, Thorell JI, Wolheim FA. Lactoferrin and lysozyme in arthritic exudates. Acta Med Scand 1977;202: 313-18.

41 Kleesiek K, Reinards R, Brackertz D, Neumann S, Lang H, Greiling H. Granulocyte elastase as a new biochemical marker in the diagnosis of chronic joint diseases. Rheumatol Int 1986;6940:161-9.

42 Adeyemi EO, Campos LB, Loizou S, Walport MJ, Hodgson HJ. Plasma lactoferrin and neutrophil elastase in rheumatoid arthritis and systemic lupus erythematosus. Br J Rheumatol 1990;29:15-20.

43 Brown RD, Yuen E, Rickard KA, Vincent PC, Young G, Kronenberg H. Plasma lactoferrin in patients with neutropenia. Blut 1986;52:289-95

44 Kremer JM. Possible mechanisms of action of methotrexate in patients with rheumatoid arthritis. $\mathrm{Br} \mathrm{J}$ Rheumatol 1995;34(suppl 2):26-9.

45 Ertug H, Arman M, Yegin O. Neutrophil, monocyte and lymphocyte locomotion in rheumatic fever and rheumatoid arthritis. Turk J Pediatr 1990;32:73-8.

46 Espersen GT, Ernst E, Vestergaard M, Pedersen JO, Grunnet N. Changes in PMN leukocyte migration activity and complement C3d levels in RA patients with high disease activity during steroid treatment. Scand J Rheumatol 1989; 18:51-6.

47 Mur E, Zabernigg A, Hilbe W, Eisterer W, Halder W, Thaler J. Oxidative burst of neutrophils in patients with rheumatoid arthritis: influence of various cytokines and medicatoid arthritis: influence of various cytokine
tions. Clin Exp Rheumatol 1997;15:233-7.

48 Storgaard M, Jensen MP, Stengaardpedersen K, Moller BK, Andersen PL, Obel N. Effects of methotrexate, sulfasalazine and aurothiomalate on polymorphonuclear leukocytes in rheumatoid-arthritis. Scand J Rheumatol 1996;25:16873.

49 Youssef PP, Cormack J, Evill CA, Peter DT, Robertsthomson PJ, Ahern MJ, et al. Neutrophil trafficking into inflamed joints in patients with rheumatoid arthritis and the effects of methyl prednisolone. Arthritis Rheum 1996;39:216-25.

50 Youssef P, Robertsthomson P, Ahern M, Smith M. Pulse methylprednisolone in rheumatoid arhthritis - effects on peripheral blood and synovial fluid neutrophil surface phenotype. J Rheumatol 1995;22:2065-71. 